\title{
Analysis on CBIR with Color and Texture-based Feature Extraction using SVM
}

\author{
Binay Kumar Yadav \\ CSE, SKIT, Jaipur \\ 302017, India
}

\author{
Neha Janu \\ CSE, SKIT, Jaipur \\ 302017, India
}

\author{
Sushila Vishnoi \\ CSE, SKIT, Jaipur \\ 302017, India
}

\author{
Vipin Jain \\ CSE, SKIT, Jaipur \\ 302017, India
}

\begin{abstract}
In this technology era, images have become a major part of information processing. An Image plays an important role in Image registration (IR) processing for the extraction of information. There are various fields like in medical, tourism and geological, weather systems forecasting used image registration. In this paper, IR is presented based on Support Vector Machine learning in the content-based image retrieval system. A Support Vector Machine (SVM) for the purpose of retrieval of images similar to the query image. Using the SVM classifier, the system can retrieve more images relevant to the query in the database efficiently. There are many traditional techniques that have been used to retrieve images. One of the Content-based image retrievals has the most popular research area in the last few years. Image retrieval is a technique of finding out the most important features of the image. The main task of content-based image retrieval (CBIR) is to get a similar images as well as perfect and fast result. In this CBIR system, effective organization of the image database used to improve the performance of the system. The study of contentbased image retrieval (CBIR) technique has become an important research issue. In this way, studied and analyzed of various features as an individual or in combinations. Through the studied of various research papers after that conclude the color and texture-based feature extraction is the most important for imparting the best extraction and support vector machine makes this task more easy and effective.
\end{abstract}

\section{Keywords}

Image Registration, CBIR, SVM, Feature Extraction, Point Cloud.

\section{INTRODUCTION}

Now a day's image processing performs a vital role in image registration. A new research field was born in the 90's: Content-based Image Retrieval aims at indexing and retrieving images based on their visual contents. It is also called Query By Image Content (QBIC), presents the technologies allowing to organize digital pictures by their visual features. They are based on the application of computer vision techniques to the image retrieval problem in large databases. CBIR consists of retrieving the most visually similar images to a given query image from a database of images. One of the main tasks of CBIR is similarity comparison.

\subsection{Image Registration Process}

Image registration is an elementary job in image processing used for pattern matching of two or more pictures taken at different time slots from different sensors and from different viewpoints. Almost all large industries which appraise images call for the image registration as a stalwartly associated operation. Literal guesses of anomalies where image registration is a major component embrace identical for objective reorganization real time images are targeted. There are two types of Image registration are performed on 3-D datasets first manually and second automatically. In manually registration human operators are responsible for all the process corresponding features of the images are to be registered. Manual process is not only monotonous and exhausting but also subject to discrepancy and limited accuracy. That's why there is a heavy standard needs to develop automatic registration techniques that require less time or no human operator control [7].

Generally there are four supporters' steps for image registration such as:

i) Feature Detection: It is an essential step for Image registration. It detect the features like closed-boundary fields, ranges, edges, outlines, line points going across, angles, and so on.

ii) Feature Matching: In this step, the letters between the features sensed in the sensed image and those sensed in the statement, direction image is got started. Different point descriptors and similarity measures in company with spatial relationships among the features are used for that purpose.

iii) Transform model Estimation: The sort and parameters of the so-named mapping purposes, uses, getting into line the sensed image with the statement, direction image, are put a value on. The parameters of the mapping purposes, uses are worked out with the help of the made certain point letters.

iv) Image re-sampling and transformation: The sensed image is greatly changed with the help of the mapping purposes, uses. Image values in non-integer orders are worked out by the right interpolation expert way of art.

\subsection{Overview of CBIR System}

Most CBIR systems are the artifacts of research, therefore focuses on a single aspect of CBIR. Two types of CBIR systems exist, one is research based CBIR systems and the other is commercial based CBIR systems is called Query based image content (QBIC). The commercial versions display more standard searching capabilities and are typically less advanced. Various CBIR systems offer user interface with flexibility that enables more powerful query design. In typical CBIR systems, the visual content of the pictures are extracted from the database. And it described by multidimensional feature vectors. These features vector of the images in the database forms a feature database. To retrieve the images, users offer the retrieval system with example images. 


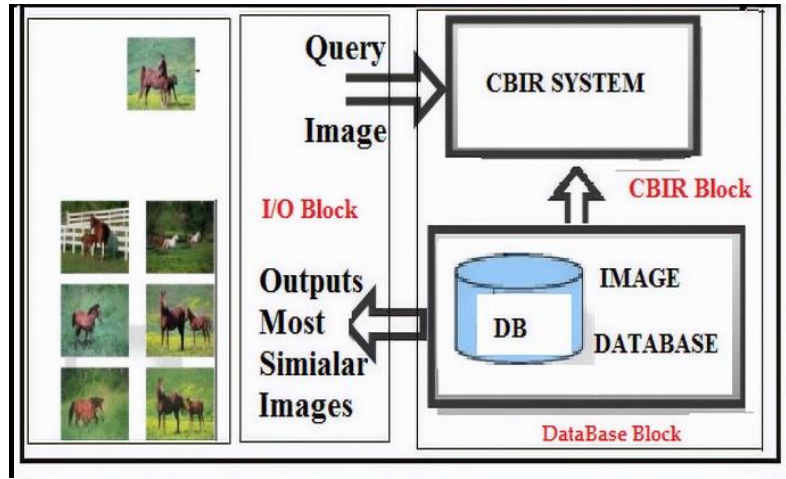

Figure [1]: Block Diagram of CBIR

There are three main components used in this CBIR system such as:

i) User's Query ii) Database Unit iii) Retrieval System i. Query unit: Query unit randomly selects an image from the database and then three features are extracted and stored as a feature vector. The three features are color, texture, and shape. The color feature used is the color moment. Extracting the color feature, first, the image is transformed into color space and then the image is partitioned into the grid. There are some moments are extracted. To extract the texture feature pyramid structured and tree-structured wavelet transform is used. The image is transformed to grayscale and Daubechies wavelet is applied. Finally, the entire features are combined from the feature vector of the query image.

\section{ii. Database unit:}

In the database unit feature vectors of all the images are extracted and stored as a feature database (FDB). For comparison query, image and database images should have similar features and they must be of the same length. So similar to the query unit Color, Texture and Shape features are extracted.

iii. Retrieval unit: This unit takes the feature vector of the query image and feature database. It then performs an SVM classification. After that, the query image is classified into any one of the class. Then it computes the similarity between the query image and the images in that class where the query image is classified and the top 20 images are retrieved

\subsection{CBIR Applications:}

There are many applications for CBIR technology as below:

- To identification of defect and fault in industrial automation.

- In medical plays a very important role in Tumors detection, Improve MRI and CT scan.

- For the weather forecast, satellite images.

- In the defense used for detection of targets.

\subsection{Feature Extraction}

The feature extraction techniques are used to extract the features. There are some features like color, texture, shape, and feature vectors etc.

a. Color:It is a property that depends on the reflection of light to the eye and the processing of that information in the brain. Generally color uses every day to tell the difference between objects, places, and the time of day. Usually, colors are defined in 3-D color spaces. Color feature extraction usages color space, color quantization \& similarity measurement key components. These could either be RGB (Red, Green, and Blue), HSV (Hue, Saturation, and Value) or HSB (Hue, Saturation, and Brightness). Color histogram moments are particular examples where color types are used to retrieve images. Color is widely used for image representation and independent of the size of an image.

$b$. Texture: Texture is that innate property of all surfaces that describes visual patterns, each having properties of homogeneity. It contains important information about the structural arrangement of the surface, such as; clouds, leaves, bricks, fabric, etc. It also describes the relationship of the surface to the surrounding environment.

c. Shape: Shape defined as the characteristic surface configuration of an object; an outline or contour. Shape does not mention to the shape of an image but to the shape of a specific region that is being sought out. It permits an object to be distinguished from its surroundings by its outline. Shape representations can be generally divided into two categories: Boundary-based, and Region-based. Shape descriptors may also need to be invariant to translation, rotation, and scale.

d. Feature Vectors: -In pattern recognition and machine learning, a feature vector is an n-dimensional vector of numerical features that represent some object. Many algorithms in machine learning require a numerical representation of objects since such representations facilitate processing and statistical analysis. When representing images, the feature values might correspond to the pixels of an image, when representing texts perhaps to term occurrence frequencies.

1.4.1 Feature Extraction Techniques: There are following techniques used for Feature extraction as follows:

1.4.1.1 Color-Based Feature Extraction: There are following Color Feature Extraction namely first one is Color moments and another one is Color Auto-Correlogram.

$a$.Color moments: Color moments are measures that characterize color distribution in an image. Each comparison between images results in a similarity score, and the lower this score is the more identical the two images are supposed to be. Color indexing (CI) is the main application of color moments. Images can be indexed, and the index will contain the computed color moments. Color moments can be computed for any color model. Three color moments are computed per channel ( 9 moments if the color model is RGB and 12 moments if the color model is CMYK). 


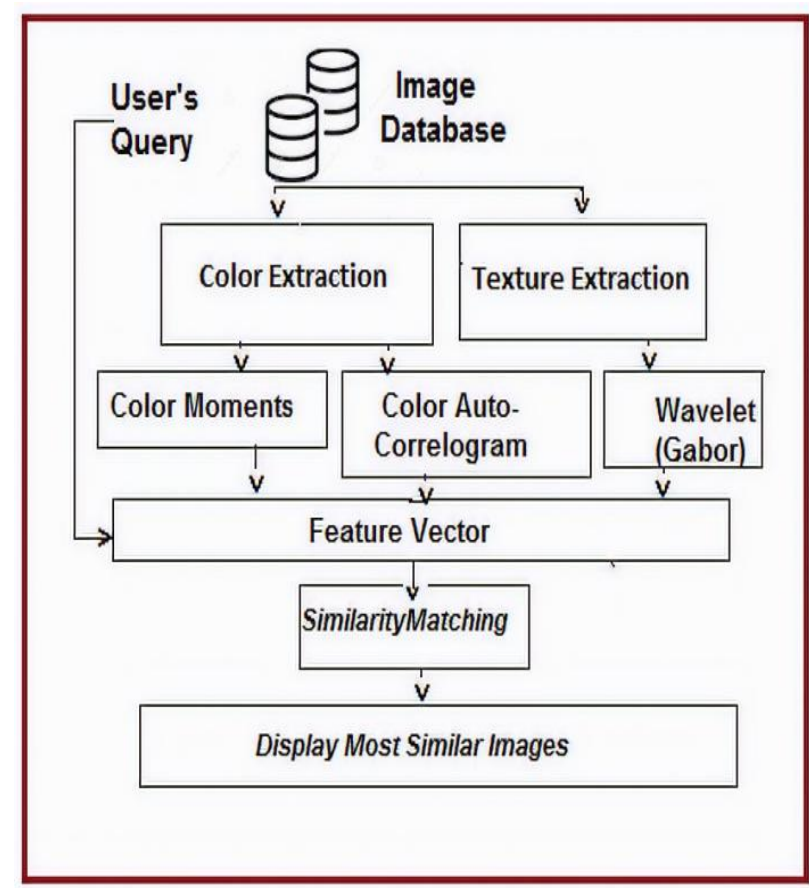

Figure [2]: Feature extraction System

b. Color Auto-Correlogram: Correlogram can be stored as a table indexed by pairs of colors (i,j) where d-th entry shows the probability of finding a pixel $\mathrm{j}$ from pixel $\mathrm{i}$ at distance $\mathrm{d}$. Whereas an auto-correlogram can be stored as a table indexed by color I where d-th entry shows the probability of finding a pixel i from the same pixel at distance " $d$ ". Hence autocorrelogram shows the spatial correlation between identical colors only [3].

1.4.1.2 Texture-Based Feature Extraction: In this Text-based technique, there is some Texture properties include including Coarseness, Contrast, Directionality, Line-likeness, Regularity, and Roughness in the image. There are some methods of classifying texture such Wavelet Transform and Gabor Wavelet.

a. Wavelet: As a mathematical tool, wavelets can be used to extract information from many different kinds of data, including audio signals and images. Wavelet analysis allows the use of long time intervals where mostly people want more precise low-frequency information. It is capable of revealing aspects of data that other signal analysis techniques miss aspects like trends, breakdown points, discontinuities in higher derivatives, and self-similarity. Wavelet analysis can often compress or de-noise a signal without appreciable degradation [10].

b. Gabor Wavelet: The Gabor filter, named after Dennis Gabor, is a linear filter used for edge detection. Gabor filter is also called Gabor wavelet). It is widely adopted to extract texture features from the images for image retrieval. Conceptually Gabor filters are a group of wavelets, with each wavelet capturing energy at a specific frequency and a specific direction. Frequency and orientation representations of the Gabor filter are similar to those of the human visual system. Gabor filters use the Fourier transform of the harmonic function and the Fourier transform of the Gaussian function for the extraction purpose. Texture features can then be extracted from this group of energy distributions. The frequency and orientation tunable property of Gabor filter makes it useful for texture analysis [5].

\subsection{Performance Evaluation:}

In CBIR system the performance of a retrieval system is evaluated based on several criteria. Some of the commonly used performance measures are average precision, average recall, average retrieval rate .All these performance measures parameter are computed using precision and recall values computed for each query image as figure below:

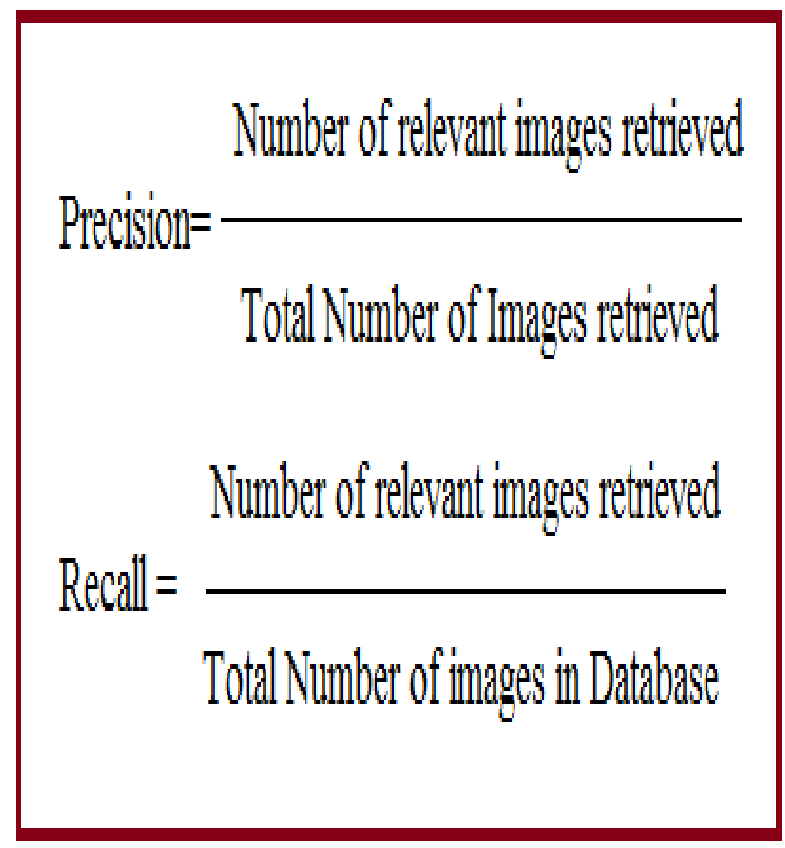

Figure [3]: Precision and Recall Calculation

\section{LITERATURE SURVEY}

In this literature survey we analyzed various research papers that described the new trends of CBIR techniques by various authors in various domains. Apart from that some technical aspects of CBIR Systems is discussed also. There are many existing technical aspects describe as below:

\subsection{Overview of Support Vector Machine (SVM)}

Support vector machine (SVM) was proposed by V. Vapnik in mid-1990.It is the most popular machine learning algorithm in the last decade. Now a day's it is widely applied and even served as the baseline in computer vision, pattern recognition, information retrieval, and data mining also.SVM is a deterministic algorithm. It is used to find the linear separating hyperplane of a binary labeled dataset. It is a classifier defined by a separating hyperplane. SVM model represents the examples as points in space, mapped, So that the examples of the separate categories are divided by a clear gap that is as wide as possible. It is used to performing linear classification and non-linear classification. It provides implicitly mapping their inputs into high-dimensional feature spaces. SVM is a binary classification method that takes as input labelled data from two classes, after that it produces the outputs as a model file for classifying new unlabeled /labelled data into one of two classes. 


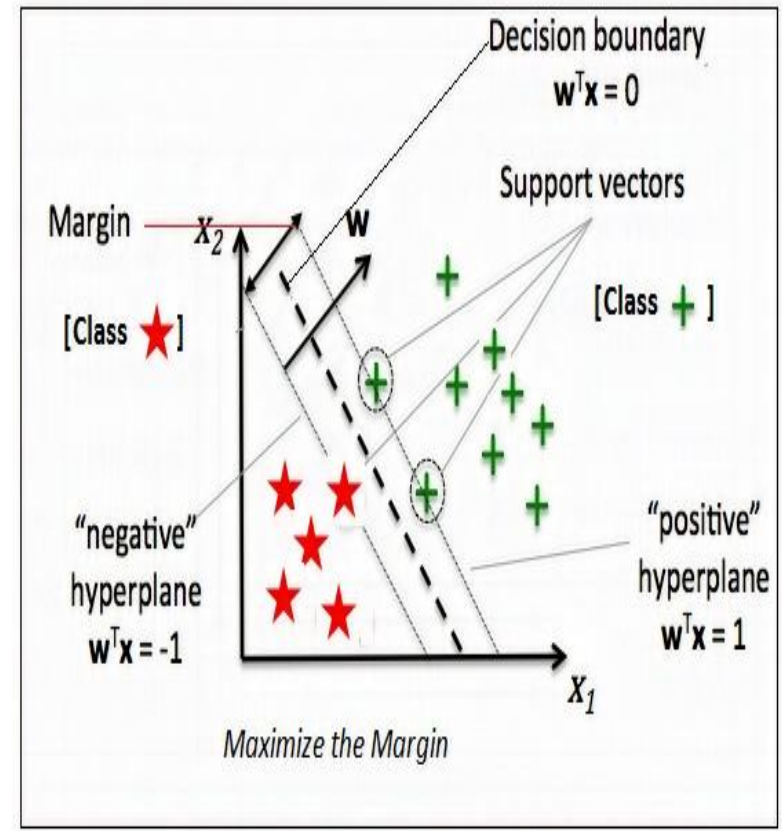

Figure [4]: SVM maximize the Hyperplane and Margin two different class Red Star and Green plus(under 2D)

The use of SVM likes machine learning technique, involves two basic steps namely training and testing. Training an SVM involves feeding known data to the SVM along with previously known decision values, thus forming a finite training set.

There are two class classification problem as input data is mapped into higher dimensional space using RBF kernel and hyper plane linear classifier is applied in this transformed space utilizing those patterns vectors that are closest to the decision boundary.

Let $\mathrm{m}$-dimensional inputs $=\mathrm{xi} \quad(\mathrm{i}=1,2,3 \ldots \ldots \ldots . \mathrm{M})$ belong to Class -“*”or Class -“+” and the associated labels be $\mathrm{yi}=1$ for Class $\mathrm{A}$ and -1 for Class B.

Here Class -A is Red Star and Class -B is Green Plus. Decision function for SVM is $\mathrm{D}(\mathrm{x})=\mathrm{w} \mathrm{T} x+\mathrm{b}$;

Where $\mathrm{w}$ is an m-dimensional vector, $\mathrm{b}$ is a scalar, and $\mathrm{yi}$ $\mathrm{D}(\mathrm{xi}) \geq 1$ for $\mathrm{i}=1,2,3 \ldots, \mathrm{M}$.

The distance between the separating hyper plane $\mathrm{D}(\mathrm{x})=0$ and the training datum nearest to the hyper plane is called the margin. The hyper plane $\mathrm{D}(\mathrm{x})=0$ with the maximum margin is called the optimal separating hyper plane.

\subsection{Surface Based Image Registration}

The 3-D edge surface of an anatomic object or composition is innate and gives major geometrical feature data that can be promoted in the medical industry for image registration. These registration methods fasten shaping corresponding surfaces in different images and computing the transformation that can be used for aligning these surfaces. Surface representation of points on the surface is just one thing that can be collected. A facial view surfaces an implicit surface or a parametric surface like a B-spline surface. Extraction of surfaces such as the skin or bone is fairly easy and head CT and MR images automatically [12].

\subsubsection{Applications of Surface-Based Image Registration}

Image registration is widely used remote sensing, medical imaging, computer vision, and in many others. Surface Based Image Registration technique is divided into following main groups such as:

a. Different Times: Images of the same view are acquired at different times. The mean is to find and estimate changes in the view.

b. Different Viewpoints: Images of the same scene are acquired from different perspectives. The aim of a large 2-D view or a 3-D representation of the scene being imaged is to gain. There are some applications like remote sensing: for mosaicing of images of the surveyed area and Computer vision for shape recovery.

c. Multimodal modal image registration (Different Sensors): Image of the same scene is acquired from a different source of sensors. The main aim is to integrate the information obtained from different source streams to gain more detailed scene representation.

\subsection{Iterative Closest Point Algorithm:}

This algorithm is used to evaluation on $3 \mathrm{D}$ medical data. There are different ICP variants can be classified according to different criteria such as Selecting subsets from the given 3D data sets, Finding correspondence points, Weighting the estimated correspondence pairs, Rejecting false matches, Assigning an error metric, Minimizing the error metric.ICP algorithm is a general-purpose, representation-independent shape-based the number on a list algorithm that can be used with a range of geometrical early persons including point puts, line part puts, triangle puts (much-sided comes to the top), and if true, then some other is necessarily true and parametric curves and comes to the top.

$$
\begin{aligned}
& \mathrm{D}(\mathrm{T}(\mathrm{X}), \mathrm{Y})= \\
& \sqrt{\sum_{j=1}^{N} W_{j}^{2}} d^{\mathbf{2}}\left(T(x \boldsymbol{j}), y=\sqrt{\sum_{j=\mathbf{1}}^{N} W_{j}^{2} \|} T(x \boldsymbol{j})-\boldsymbol{y} \boldsymbol{j} \|^{2} \mathbf{- 3 . 1}\right. \\
& \text { Where } \quad \mathrm{yj}=\mathrm{C}(\mathrm{T}(\mathrm{xj}), \mathrm{y}) \quad-\quad 3.2
\end{aligned}
$$

\subsection{Cademic Proposals:}

Literature survey is most important for understanding, extracting and gaining much more knowledge about specific area. In this paper many existing proposals studied to image retrieval as:

M.E.ElAlami et.al (2011)[1] They have discussed the 3D color histogram and the Gabor filter algorithm is able to effectively describe various properties of the image. A new model integrated the color coherence vector and wavelets features to enhance retrieval performance. There are two sequential strategies performed, preliminary and deeply reduction, for extracting the most relevant features set. After that this strategy is used to reduce the search space and consumed time in the retrieval process.

Neha Janu et.al (2017)[4] that presented the feature extraction technique in the frequency domain. There are three feature extraction technique used likes Gabor filter, Discrete Wavelet Transform and Discrete Cosine Transform.

B.Bohra et.al (2014)[5] that presented in this paper, to minimization of time and error in the surface based image registration process of 3-D data sets using Point Cloud data structure. Normally used in the medical industry to store the CT images, MRI images and Tumor images and also used to 
make 3-D models of real objects, which is the best suitable data structure for the image registration process of 3-D data sets. I.C.P algorithm which registers two 3-D data sets and find the closest points into data sets as per giving tolerance distance.

N. Ali, et.al (2016)[6] that presented in this paper, to enhances the performance of image retrieval. The Standard images are constructed by following the rule of thirds that divide an image into nine equal parts by placing objects or regions of interest at the intersecting lines of the grid. So, color and texture feature gives an efficient and appropriate result in the human visual system.

Neha Janu et.al (2017), [7] that presented the Gabor feature extractions have higher accuracy compared to discrete wavelet transform and discrete cosine transform. The analysis of the better result of Gabor features, it is analyzed that Gabor is better extraction technique for edge or shape features extraction compared to DWT and DCT feature extraction. In DWT and DCT feature extraction, the feature is extracted from Low-frequency feature sub band (LL) and other frequency band are discarded so some of the features are lost which is the cause of lower accuracy compare to Gabor. Gabor with scale projection achieved better accuracy compared to Gabor without scale.

G.S.Somnugpon et.al (2016)[12] that presented the combination of two new methods likes image texture and spatial correlation of pairs of color in feature provides more robustness to the high changing image. To give averaging precision also better the existing methods. By using color correlogram will treat information about spatial color correlation, while EDH provides the geometry information in the case of the same image but different color. It is a combination of low-level features gives a better result than an individual feature. Euclidean distance is used for similarity measurement.

Atif Nazir et.al (2018) [14] .In this paper they presented the Global feature includes shape descriptors, contour representations, and texture features. CBIR strategy combines the local and global features for low-level information. In this paper, they have proposed a new CBIR technique to fuse color and texture features. Color Histogram $(\mathrm{CH})$ is used to extract color information. Texture features are extracted by Discrete Wavelet Transform (DWT) and Edge Histogram Descriptor (EDH). The features are created. They have combined two or more features give better outputs as compared to one feature. So, color and texture feature gives an efficient and appropriate result in the human visual system. RuigangFu et.al (2016)[16] they have provided by mainly features representations and similarity measures. This paper applies convolution neural network (CNN) to CBIR and uses linear support vector machine (SVM) to train a hyperplane which can separate similar image pairs and dissimilar image pairs to a large degree. Experiments show that the method can significantly improve the performance of CBIR for object image retrieval tasks. This Research paper a CBIR system based on CNN and SVM, where CNN is used to extract the feature representations and SVM is used to learn the similarity measures.

\section{CONCLUSIONS AND RESEARCH DIRECTIONS}

In this paper discussed brief summary of image retrieval and its classifier support vector machine (SVM). Many researchers have been implemented many feature extractions techniques to retrieve the images from the training database. Each of these databases includes a variety of different images with different colors, shapes, and textures. Color histogram (RGB and HSV) is used as a feature extraction technique for retrieving images based on color . The Gabor filters are found to be best for implementing texture-based feature extraction. For similarity measurements, Euclidean distance and weighted Euclidean distance can be used. For a performance measurement precision and recall rate can be used .After that seen in previous work that the performance is based on color and texture separately as well as combination of color and texture both. In this paper analysis, finally found and conclude that the results of image retrieval using the individual feature color or texture are not as good as using combining these features. After combining the important subfeatures of color, texture, and shape, the result is improved and the efficiency of image retrieval is better. At last, the based on the study of various papers it is concluded that support vector machine makes this task more easy and effective. In future Surface-based image registration techniques are widely used in 3-D Data sets image registration in various industries. The most efficient algorithm in the entire surface-based image registration technique with Iterative Closest Point (ICP).ICP is used for better image registration. In future directions, image registration in 3-D datasets can be done with SVM and ICP.

\section{ACKNOWLEDGMENTS}

The acknowledgment is addressed to Swami Keshvanand Institute of Technology, Management \& Gramothan (SKIT), Jaipur for supporting with us. The authors want to thank all the researchers for their previous work.

\section{REFERENCES}

[1] M. E. Elalami, A novel image retrieval model based on the most relevant features, Knowledge-Based Syst., vol. 24, no. 1, pp. 23-32, 2011.

[2] Vandana Vinayak and Sonika Jindal ,'CBIR System using Color Moment and Color Auto-Correlogram with Block Truncation Coding", International Journal of Computer Applications , March (2017)161(9):1-7.

[3] J. Yue, Z. Li, L. Liu, and Z. Fu, Content-based image retrieval using color and texture fused features, Math. Comput. Model., vol. 54, no. 34,pp. 1121-1127, 2011.

[4] Neha, Pratistha Mathur, "Three Level Optimization Model of Scale Gabor Features for Facial Expression Recognition", International Journal of Engineering and Technology (UAE), ISSN No. 2227-524X, Volume-7, Issue-2.24, Feb 2018, pp 348-350.

[5] Brahmdutt Bohra1\# Deepak Gupta2* Shikha Gupta3\#” An Efficient Approach of Image Registration Using Point Cloud Datasets".

[6] Nouman Ali, Khalid Bashir Bajwa , Robert Sablatnig, Zahid Mehmood " Image retrieval by addition of spatial information based on histograms of triangular regions".

[7] Janu Neha, Pratistha Mathur, "Performance analysis of frequency domain based feature extraction techniques for 
facial expression recognition." In 7th International Conference on Cloud Computing, Data Science \& Engineering-Confluence, 2017, pp. 591-594. IEEE, 2017.

[8] Jan Elseberg, Dorit Borrmann and Andreas Nüchter, "One billion points in the cloud - an octree for efficient processing of 3D laser scans". In Proc. ISPRS Journal of Photogrammetry and Remote Sensing 76 (2013) 76-88.

[9] Brian Amberg, Sami Romdhani and Thomas Vetter "Optimal Step Nonrigid ICP Algorithms for Surface Registration". This work was supported in part by Microsoft Research through the European PhD Scholarship Programme.

[10] J. Yu, Z. Qin, T Wan, and X. Zhang, Feature integration analysis of bag-of-features model for image retrieval, Neurocomputing, vol. 120, pp. 355-364, 2013.

[11] T. Kato, "Database architecture for content-based image retrieval", in Image Storage and Retrieval Systems, Proc SPIE 1662, (1992) pp112-123.

[12] S. Somnugpong and K. Khiewwan, "Content Based Image Retrieval using a combination of Color
Correlograms and Edge Direction Histogram", 13th International Joint Conference on Computer Science and Software Engineering,DOI:10.1109, IEEE, (2016).

[13] C. S.Won, D. K. Park and Y. S. Jeon, "an efficient use of MPEG-7 Color Layout and Edge Histogram Descriptors", proceeding of the ACM workshop on multimedia, (2000), pp. 51-54.

[14] Atif Nazir, Rehan Ashraf, Talha Hamdani, Nouman Ali,"Content based image retrieval system by using HSV color histogram, discrete wavelet transform and edge histogram descriptor",International Conference on Computing, Mathematics and Engineering Technologies (iCoMET),Azad Kashmir,(2018)1-6.

[15] Neha Janu, Pratistha Mathur, "Performance Analysis of Feature Extraction Techniques for Facial Expression Recognition", International journal on Computer Applications, ISSN No. 0975 -8887, Volume-166, Issue1, May 2017.

[16] Ruigang Fu, Biao Li, Yinghui Gao, Ping Wang," Content-Based Image Retrieval Based on $\mathrm{CNN}$ and SVM", 2016 2nd IEEE International Conference on Computer and Communications, pages (638-642). 